\title{
The stellar and gaseous kinematics in NGC 253
}

\author{
F. Prada ${ }^{1}$, C.M. Gutiérrez ${ }^{1}$, C. D. McKeith ${ }^{2,3}$
}

Received —

\footnotetext{
${ }^{1}$ Instituto de Astrofísica de Canarias, 38200 La Laguna, Tenerife, SPAIN, (http://www.iac.es). E-mail: fprada@iac.es, cgc@iac.es

${ }^{2}$ Department of Pure and Applied Physics, Queen's University of Belfast, Belfast BT7 1NN, UK

${ }^{3}$ Deceased
} 


\begin{abstract}
This paper presents observations with intermediate spectral and spatial resolution along the major and minor axes of the starburst galaxy NGC 253. The spectral ranges analyzed are in the region of the stellar $\mathrm{MgI} b(\sim 5175 \AA)$, the near IR CaII triplet $(\sim 8550 \AA)$ absorption features, and the region of the $\mathrm{H} \alpha$ emission line. We have compared the shape of the stellar features with those of reference stars and determined the line-of-sight velocity distribution (LOSVD) of the stellar component by using a two-dimensional Gaussian decomposition algorithm, and show for the first time the rotation curve of the stellar component in NGC 253. Comparing the recesion velocity curves of the gas and stars, it is seen that the stellar component has a decoupled kinematics respect to the gas, displaying a shollower velocity gradient and larger velocity dispersion that the gas in the inner regions. The minor axis kinematics together with the kinematics across the central $40^{\prime \prime}$ along the major axis, suggest the presence of a rotating body with a kinematically misaligned axis respect to the main disk of the galaxy. The asymmetries in the LOSVD along the minor axis together with the steep velocity gradient of the gaseous component suggest a merger scenario to explain the these kinematically signatures. The enclosed mass in the central regions is computed to be $(2.4 \pm 0.5) \times 10^{7} \mathrm{M}_{\odot}$ for a radius of $r=0.7^{\prime \prime}$ (10 pc). A double gaseous component in the central 6 arcsecs is detected from the [SIII] $\lambda 9069 \AA$ data along the minor axis; this seems to be the signature of a superbubble due to a supernova rate of $0.05 \mathrm{yr}^{-1}$.
\end{abstract}

Subject headings: galaxies: individual (NGC 253) — galaxies: kinematics and dynamics — galaxies: spiral — galaxies: starburst — dust, extinction 


\section{INTRODUCTION}

The well known starburst galaxy NGC 253 (e.g. Rieke et al. 1980), classified as type SAB(s)c (de Vaucouleurs et al. 1991) has been subject of many detailed kinematical studies of its gaseous component. These studies include long-slit optical spectroscopy (Ulrich 1978; Muñoz-Tuñon,Vilchez \& Castañeda 1993; Arnaboldi et al. 1995, hereinafter ARN95), which shows large deviations from non-circular motions. These deviations have been attributed to the outflow of gas due the presence of massive star formation (e.g. Heckman et al. 1990), as well as the presence of heavy obscuration in the central regions (Scoville et al. 1985; Sams et al. 1994). The large amount of extinction seems to be the reason for the steeper rotation curve obtained with near-IR emission lines compared to optical lines (Puxley \& Brand 1995; Prada et al. 1996). The infrared measurements are in agreement with the ${ }^{12} \mathrm{CO}(\mathrm{J}=1-0)$ velocity field derived by Canzian et al. (1988). The most complete analysis of the gaseous component in the innermost regions has been done using the radio recombination H92 $\alpha$ line by Anantharamaiah \& Goss (1996) (hereinafter AG96). They determined a velocity field in the central $150 \mathrm{pc}$ displaying a distinct gaseous kinematics subsystem, exhibing rotation in a plane perpendicular to the galactic disk, and an inner region with possible counterrotation in the plane of the disk. The authors suggest that this result may be indicative of a secondary bar inside the known primary bar, or alternatively, a signature of a merger or an accretion event during the history of the galaxy.

The analysis and comparison of the stellar kinematics with the gaseous motions would be of great interest in determining the nature of this peculiar kinematics found in the central regions of the galaxy. However, up to now the information on the stellar kinematics of NGC 253 is poor and only the velocity dispersion in the nucleus is known (Lutz \& Prada 1995; Oliva et al. 1995). The study presented in this paper reports the results of spectroscopic observations taken in the region of the MgIb, the CaII IR triplet stellar features, and the $\mathrm{H} \alpha$ emission line along the major and minor axes of NGC 253.

The paper is structured as follows: Section 2 presents the observations and data reduction, Section 3 analyzes the gaseous kinematics and the starbust in the center of the galaxy, and Section 4 determines the stellar kinematics and evaluates the enclosed mass in the nuclear region. Section 5 is dedicated to the comparison between the stellar and gaseous kinematics. 


\section{OBSERVATIONS AND DATA REDUCTION}

During several campaigns, we obtained long-slit spectra along the major (p.a. $52^{\circ}$ ) and minor (p.a. $321^{\circ}$ ) axes of NGC 253 with the double arm ISIS spectrograph on the $4.2 \mathrm{~m}$ William Herschel Telescope at the Observatorio del Roque de los Muchachos, La Palma, Spain. We used a $1124 \times 1124$ Tek CCD with $0.36^{\prime \prime}$ pixel $^{-1}$ in the red and blue arm of ISIS respectively. The spectral dispersion was $0.78 \AA$ per pixel (with a FWHM=2 pixels); CuAr and CuNe lamps were used for spectral calibration. The observations are summarized in Table 1. In the observations of November 1995, the slit was $0.9^{\prime \prime}$ wide, slightly undersampling the $\sim 0.9^{\prime \prime}$ seeing, and was placed along the major axis centered on the apparent optical nucleus. In the observation of July and August 1996 the slit was placed at the dynamical center given by AG96 with a slit width of $1.3^{\prime \prime}$ and seeing of $\sim 1.2^{\prime \prime}$. The slit was $4^{\prime}$ long in all the runs. In each of these runs, standard stars with spectral types ranging from G5III to K4III were also observed. These stars were used as templates to determine the stellar line of sight velocity distribution (LOSVD) of NGC 253 as it will be explained in next sections. Standard data reduction including bias subtraction, flat field correction and wavelength calibration was carried out using the IRAF package. The sky lines were subtracted using template spectra from the ends of the slit. A distance of 3.4 Mpc (Sandage \& Tammann 1975) for NGC 253 was adopted, yielding a scale of $16.5 \mathrm{pc} \operatorname{arcsec}^{-1}$.

\section{THE GASEOUS KINEMATICS AND STAR FORMATION ACTIVITY}

Figure 1 shows the gaseous position-velocity curves along the major axis of the galaxy, determined by fitting Gaussian profiles to the [NII] $\lambda 6584 \AA$ and the [SII] $\lambda \lambda 67176731 \AA \mathrm{H} \alpha$ emission lines. The radial velocities are heliocentric and the zero position for the spatial axis comes from the continuum peak at $\sim$ $6500 \AA$ A. The systemic velocity of $V_{\mathrm{HEL}}=230 \pm 10 \mathrm{~km} \mathrm{~s}^{-1}$ is in agreement with previous measurements (e.g. ARN95). The results for the three species are in agreement and show that the center of symmetry for the gas rotation curve is shifted $11^{\prime \prime} \mathrm{SW}$ from the continuum peak as was first reported by ARN95. Figure 2 compares the [SIII] $\lambda 9069 \AA$ velocity curve (measured in November 1995) with the near-IR $\mathrm{H}_{2} \lambda=2.1218$ $\mu \mathrm{m}$ position-velocity curve determined by Prada et al. (1996), and with recent measurements by AG96

\footnotetext{
${ }^{4}$ IRAF is distributed by National Optical Astronomical Observatories, which is operated by the Association of Universities for Research in Astronomy, Inc., under contract with the National Science Foundation
} 
using the radio recombination line $\mathrm{H} 92 \alpha$. There is a good agreement between the three species except for the SW of the nucleus suggesting that this side is especially dusty. This velocity difference has been also found by Prada et al. (1996) comparing near-IR data with optical data, and was attributed to an optical depth effect due to dust extinction (see Section 5).

Figure 3 compares the minor axis position-velocity curve of the [SIII] emission line with the H $\alpha$ data by Ulrich (1978) and Heckman et al. (1990), as well as for the measurements by AG96 using H92 $\alpha$. The [SIII] spectra show two components in the central $6^{\prime \prime}$ : the brighter component has the systemic velocity $\left(\mathrm{V}_{H E L}=230 \mathrm{~km} \mathrm{~s}^{-1}\right)$ while the fainter one is blueshifted $140 \mathrm{~km} \mathrm{~s}^{-1}$. Further out from the center, our data agree with the Ulrich (1978) data, whilst the data by Heckman et al (1990) given only for the SE, show two components beyond $10^{\prime \prime}$ from the center. We believe that the deeper penetration through the dust of the near-IR wavelengths allow us to see into the equator of the galaxy the two components that Heckman et. al (1990) and Schulz \& Wegner (1992) attribute to a starburst-driven superwind at the surface of a cone or bubble. However, our [SIII] data do not agree with the very steep H $92 \alpha$ velocity gradient reported by AG96 in the inner $4^{\prime \prime}$ (see Figure 3); this will be discussed in detail in Section 5.

Figure 4 presents the velocity dispersion curve along the major axis of NGC 253 for the optical and the [SIII] $\lambda 9069 \AA$ emission lines. The optical velocity dispersion curve shows a depression with a minimum value of $65 \mathrm{~km} \mathrm{~s}^{-1}$ at the dynamical center (11" from the continuum peak), and reaches $\sim 100 \mathrm{~km} \mathrm{~s}-1$ at $10^{\prime \prime}$ at either side of this. For the [SIII], the velocity dispersion reaches a value of $85 \mathrm{~km} \mathrm{~s}^{-1}$ at $3.5 \mathrm{SW}$ from the continuum peak and decreases slowly towards larger radii. The value of the velocity dispersion at the dynamical center is in agreement with the measurements by Oliva et al. (1996) using Br $\gamma$ for a central aperture of $4^{\prime \prime} \times 4^{\prime \prime}$.

The two components present in the [SIII] emission lines in the central $4^{\prime \prime}$ along the minor axis of NGC 253, can be interpreted as a large-scale expansion or superbubble due to supernova (SN) events. The measurement of the gas expansion velocity at the position where the slit interset the disk of the galaxy, namely the expansion velocity of the superbubble, can be used to constrain the SN rate in the nuclear regions. Colina \& Pérez-Olea (1992) consider a SN rate in the nuclear region of $0.05 \mathrm{yr}^{-1}$, in agreement with the value obtained by Heckman et al. (1990). Taking our measurements, the expansion velocity is $\sim$ $75 \mathrm{~km} \mathrm{~s}^{-1}$ deprojected using an inclination of $78.5^{\circ}$ (Pence 1981) and the density of the medium in the bubble, $\mathrm{n}_{o}=630 \mathrm{~cm}^{-3}$ (Heckman et al. 1990), we estimate a radius of $130 \mathrm{pc}$ for the superbubble in the disk of the galaxy. The radius can be estimated from the equations given by McLow \& McCray (1988) 
for the mechanical luminosity of a superbubble $L_{S N} \sim 3.6 \times 10^{29} n_{o}\left(\mathrm{~cm}^{-3}\right) R^{2}(p c) V^{3}\left(\mathrm{~km} \mathrm{~s}^{-1}\right)^{3}$. As the superbubble should be located interior to the edge-on nuclear ring of $20^{\prime \prime}$ (330 pc) in diameter reported by ARN95, this introduces a constrain in in the SN rate with values much smaller than the one derived by by Rieke et al. (1988) and Forbes et al. (1993) who estimates a SN rate of 0.1 or $0.2 \mathrm{yr}^{-1}$. For this reason we believe that the average $\mathrm{SN}$ rate in the central $300 \mathrm{pc}$ is $\sim 0.05 \mathrm{yr}^{-1}$. The value of $0.1-0.2 \mathrm{yr}^{-1} \mathrm{could} \mathrm{be}^{-}$ more local since it was determined from the presence of few radio sources (Rieke et al. 1988), and the [FeII] luminosity in the central 50 pc (Forbes et al. 1993) of the galaxy. Finally, we would like to mention that all the authors agree for a SN rate of $\sim 0.3 \mathrm{yr}^{-1}$ in the nucleus of M82.

\section{THE STELLAR KINEMATICS AND ENCLOSED MASS}

We have determined the LOSVD along the major and minor axis of NGC 253 from the long-slit MgIb (5175 $\AA$ ) and CaII IR triplet (8550 ̊) absorption spectra by means of a two-dimensional unresolved Gaussian decomposition algorithm. This method has been applied succesfully to determine the stellar kinematical structure of NGC 7331 (see Prada et al. 1996). Basically, this approach considers that the spectrum of the galaxy in a given angular position is the result of a mean stellar spectrum broadened by the internal kinematic structure of the galaxy and by the observational-experimental configuration. The intrinsic broadening represents the convolution of this mean stellar spectrum with the LOSVD. Additionally the instrumental configuration (slit-width, etc), and the atmosphere (seeing) sliglthly broaden the spectrum in the plane of the detector, introducing correlations between neighbouring pixels along both the spectral and the spatial axes respectively. We parametrize the LOSVD as a sum of two-dimensional Gaussians in the plane velocity-angular position. The dispersion of the Gaussians reflect the resolution in both axes, i.e. spectral and spatial resolution respectively. The dispersions used for the observations presented here, were $\sim 2$ pixels and $\sim 1.5$ pixels in the spectral and spatial axes respectively, with relative separation 3 and 2 pixels. The algorithm makes a two-dimensional $\chi^{2}$ minimization of the difference between the observed and the modelled spectra using a Netwon-Raphson iterative scheme. With respect to the previous algorithm by Kuijken \& Merrifield (1993), the two-dimensional decomposition takes into account the correlation between adjacent angular positions mentioned above. The use of this additional information produces little change in regions of moderate o high signal to noise ratio (SNR) but might be important in regions far away of the galactic center.

The spectral regions analyzed were $5120-5290 \AA$ and $8450-8700 \AA$ along the major and minor axes. 
As velocity template for the November 1995 observations, the K0 giant HR218 star was used. The K4III HD 213947 and the G5III HD4388 stars were used for the July and August 1996 runs respectively. Figure 5 shows the spectra in several positions along the major axis in the region of the stellar MgIb feature. The emission feature at $\sim 5200 \AA$ corresponds to the gaseous line $[\mathrm{NI}] \lambda \lambda 5198.55200 .7 \AA$. Also plotted are the modelled spectra obtained by convolution of the derived LOSVD with the spectrum of the velocity template (excluding the $[\mathrm{NI}]$ feature). Several absorption features including the main components of the $\mathrm{MgI} b$ are reconstructed indicating the reability of the algorithm, and that the stellar reference spectra is a good representation of the mean stellar spectrum in the galaxy. Figures 6 and 7 show the equivalent plots in the region around the CaII IR triplet along the major and minor axes respectively. In the case of the major axis, the spectra correspond to the August 1996 run. In both cases the CaII line at $8662 \AA$ has been excluded from the fit because it appears strongly contaminated by sky emission. The reconvolved spectra are very similar using template stars of types from G5III to K4III. Exhaustive analyses of the performance of the algorithm, including Monte Carlo simulations will be presented in a forthcoming paper (Gutiérrez \& Prada 1997).

The LOSVD along the major axis obtained from the MgIb and CaII IR triplet are symmetrical and show a single component. Figure 8 shows the radial velocities along the major axis; these have been obtained by fitting a single Gaussian to the LOSVD. These radial velocities are heliocentric and the zero position have been chosen from the continuum peak in each case. The MgI $b$ velocity curve goes from $-90^{\prime \prime}$ to $+120^{\prime \prime}$ from the continuum peak. Overplotted is a linear fit which reproduces adequately the general trend of the curve and which has a velocity gradient of $1.7 \mathrm{~km} \mathrm{~s}^{-1} \operatorname{arcsec}^{-1}$. Separation of this trend seems to occur in the region from $\sim+40^{\prime \prime}$ to $+90^{\prime \prime}$. In the case of the CaII triplet, the data of August 1996 allow to follow the velocity curve in a large spatial range (from $-25^{\prime \prime}$ to $+25^{\prime \prime}$ from the continuum peak). Also shown is a fit to the CaII data of a circular velocity motion (see Bertola et al. 1991). The CaII velocity curve shows a steeper gradient compared to the MgIb curve for the SW side probably due to the presence of dust in the SW side of the nucleus (Scoville et al. 1985; Sams et al. 1994).

Figure 9 presents the CaII IR triplet velocity curve for the minor axis obtained by fitting a single Gaussian to the LOSVD. The curve shows a velocity gradient which is not expected from circular motions along the main plane of the galaxy. Furthermore, the LOSVD shows some degree of asymmetry towards the blueshift in the inner $10^{\prime \prime}$, as it is shown in Figure 10.

Figure 11 shows the stellar velocity dispersion curve from the CaII IR data along both axes. The 
velocity dispersion in the major axis has a value of $90 \pm 5 \mathrm{~km} \mathrm{~s}^{-1}$ at $5^{\prime \prime} \mathrm{SW}$ from the continuum peak which is in agreement with the values that Oliva et al. (1996), and Lutz \& Prada (1995) give using the CO $2.3 \mu \mathrm{m}$ absorption feature for a central aperture of $4^{\prime \prime} \times 4^{\prime \prime}$. Beyond $\sim 10^{\prime \prime}$ the velocity dispersion decreases down to $70 \mathrm{~km} \mathrm{~s}^{-1}$. The velocity dispersion along the minor axis reaches a value of $110 \pm 20 \mathrm{~km} \mathrm{~s}^{-1}$ in the central $10^{\prime \prime}$. This can be attributed to the presence of two kinematically distinct components in the inner regions as revealed by the LOSVD.

Assuming that the velocity dispersion accounts for all the gravitational support (see McGinn et al. 1989) and following the notation of Gaffney et al. (1993) the enclosed mass for a given radius is

$$
M\left(r \leq r_{o}\right)=\frac{r \sigma^{2}(r)(A+B+C)}{G} .
$$

where $A=-d \ln n(r) / d \ln (r), B=-d \ln \sigma^{2}(r) / d \ln r$, and $C=V^{2}(r) / \sigma^{2}(r)$. To determine $A$ we have adopted a surface brightness profile $\mu(r) \propto r^{-0.8 \pm 0.2}$, valid for $1^{\prime \prime} \leq r \leq 5^{\prime \prime}$ determined from near-IR observations (Sams et al. 1994). Hence, we can obtain the flux density $F(r) \propto r^{1.5}$ and therefore $n(r) \propto r^{-1.5}$. $B$ and $C$ were determined from the results shown in Figures 8 and 11 . Figure 13 shows the enclosed mass at different radii: for $r=0.7^{\prime \prime}(10 \mathrm{pc})$, we obtained an enclosed mass of $(2.4 \pm 0.5) \times 10^{7} \mathrm{M}_{\odot}$. This value is in agreement with the enclosed mass found by Gaffney et al. (1993) for M82: $M(r \leq 7.5$ $\mathrm{pc})=(3 \pm 1) \times 10^{7} \mathrm{M} \odot$.

\section{THE DICHOTOMY OF THE STELLAR AND GASEOUS KINEMATICS}

The direct comparison of our measurements of the optical stellar MgIb and gaseous (e.g. H $\alpha$ ) position-velocity curves reveals a clear dichotomy between the kinematics of the gas and the stars in the central 650 pc of NGC 253 (see Figure 12). The effects of dust extinction in determining the line-of-sight velocities are very small for the two spectral lines mentioned above to explain such velocity differences between gas and stars (see Prada 1995). However, in the SW side of the nucleus, the heavy obscuration $\left(\mathrm{A}_{V} \sim 30 \mathrm{mag}\right)$ reveals a steeper velocity gradient for the stellar CaII near-IR feature, which is shallower than the $\mathrm{H} \alpha$ velocity gradient, the [SIII] line, the near-IR emission lines (Puxley \& Brand 1995; Prada el at. 1996), and the radio emission lines (AG96) in this region, but steeper than the MgIb velocity curve.

Adopting the larger wavelength velocity data, i.e. the CaII IR triplet absorption feature and the emission $\mathrm{H} 92 \alpha / \mathrm{H}_{2} \lambda 2.13 \mu \mathrm{m}$ lines as the intrinsic (i.e. extinction free) stellar and gaseous kinematics of NGC 253, we conclude that the motions of the stars in the central regions are not coupled with the gas. 
We think that the velocity difference could be a consequence of the distribution of the gas in a ring. This seems to be confirmed by the high resolution measurements made by ARN95 who claim the existence of an edge-on nuclear ring due to the presence of an Inner Linblad Resonance at $300 \mathrm{pc}$ from the center. They believe that the bar is the responsible for the gas fueling of the nuclear starburst, and therefore will influence the dynamics of the gas in the central regions. This suggests that the gas is distributed in a ring with ongoing star formation and that the stars will trace the kinematics of the regions inside the ring. This is reinforced by the larger value of the stellar velocity dispersion with respect to the gas: 90 and $65 \mathrm{~km}$ $\mathrm{s}^{-1}$ respectively. This difference also occurs is other galaxies as reported by Terlevich et al. (1990) from observations of the CaII IR triplet. They find that the only objects which have emission lines substantially narrower than absorption lines are starburst galaxies. Similar values than for NGC 253, have been found in the nucleus of M82 (Gaffney et al. 1993); in this galaxy, also the slope of the stellar velocity curve for the central regions is shallower than the ionized gas as reported by Gaffney et al. (1995). These authors conclude that the stellar peak in M82 may be an older bulge population (Gaffney \& Lester 1992; Gaffney et al. 1993), which might also be the case of NGC 253 given the remarkably similarity with the dynamics of M82.

The kinematics along the minor axis of NGC 253 reveals the presence of an ordered motion which appears well described by a circular motion with $\Delta \mathrm{V}_{\max } \sim 40 \mathrm{~km} \mathrm{~s}^{-1}$ (see Figure 9). The minor axis kinematics together with the kinematics across the central $40^{\prime \prime}$ along the major axis which has $\Delta \mathrm{V}_{\max } \sim 70$ $\mathrm{km} \mathrm{s}^{-1}$ suggest the presence of a central rotating body with a misaligned axis respec to the main rotation axis of the galaxy. According to the shape of the LOSVD, this body might contains a decoupled kinematical component in its center probably due to the accretion of material, either in the form of stars or gas which tiggered the starburst.

The counterrotation reported by AG96 in the inner 2" along the major axis is also present in our [SIII] data, although there is a velocity discrepancy in the SE regions due to dust extinction as discussed in Section 3. The fact that our [SIII] data match the H92 $\alpha$ velocity data along the major axis, suggests that dust extinction is not very high in the inner $4^{\prime \prime}$ (see Figure 2). Therefore, we do not understand, if this is the case, the very step velocity gradient found by AG96 along the minor axis. Furthermore our [SIII] minor axis data show two velocity components. We believe that the discrepancy between the two data sets can be interpreted as follows: by fitting a single Gaussian to the [SIII] emission lines we can match the H92 $\alpha$ data by shifting $3.5^{\prime \prime} \mathrm{NE}$ our data. This would suggest that the H92 $\alpha$ velocity gradient reported by AG96 might represent an artefact due to the outflow of gas. On the other hand, it could well happen that we placed the 
slit not exactly at the dynamical center, since our positioning error was of $1.25^{\prime \prime}$, and the rapid rotation seen in the H92 $\alpha$ data is a kinematic subsystem that might have the same origin than the structure seen in the stellar LOSVD along the minor axis.

This research is based on observations obtained at the WHT, operated by the ING at the Observatorio del Roque de los Muchachos at La Palma of the IAC. We thank E. Perez and R. F. Peletier for helpful discussions. The spectroscopic observations were performed on service nights in November 1995 and July and August 1996.

This paper is dedicated to the memory of C. D. McKeith, who contributed much to this work before his untimely death on Summer 1996. 


\section{REFERENCES}

Anantharamaiah, K. R., \& Goss, W. M., 1996, ApJ, 466, L13

Arnaboldi, M., Capaccioli, M., Cappellaro, E., Held,E.V., \& Koribalski,B. 1995, AJ, 110, 199

Bertola, F., Bettoni, D., Danziger, J., Sadler, E., Sparke, L., \& de Zeeuw, T. 1991, ApJ, 373, 369

Canzian, B., Mundy, L., \& Scoville, N. Z. 1988, ApJ, 333, 157

Colina, L., \& Pérez-Olea, D. 1992, MNRAS, 259, 709

de Vaucouleurs, G.,de Vaucouleurs, A., Corwin, H. G.,jr, Buta, R. J.,Paturel, G., \& Fouqué, P. 1991, Third Reference Catalogue of Bright Galaxies, Springer Verlag

Forbes, D. A.,Ward, M. J.,Rotaciuc, V.,Blietz, M.,Genzel, R., Drapatz, S.,van der Werf, P. P., \& Krabbe, A. 1993, ApJ, 406, L11

Gaffney, N. I., \& Lester, D. F. 1992, ApJ, 394, 139

Gaffney, N. I., Lester, D. F., \& Telesco, C. M. 1993, ApJ, 407, L57

Gaffney, N. I., Lester, D. F., \& Doppmann, G. 1995, PASP, 107, 68

Gutiérrez, C. M., \& Prada, F., 1997, in preparation

Heckman, T.,Armus, L., \& Miley, G. 1990, ApJS, 74, 833

Kuijken, K. \& Merrifield, M. R., 1993, MNRAS, 264, 712

Lutz, D., \& Prada, F. 1995, IAU Symposium 171, in New light on Galaxy Evolution. Heidelberg

Mac Low, M.-M., \& McCray, R. 1988, ApJ, 324, 776

McGinn, M. T.,Sellgren, K.,Becklin, E. E., \& Hall,D. N. B. 1989, ApJ, 338, 824

Munõz-Tunõz, C., Vilchez, J. M., \& Castanẽda, H. 1993, A\&A, 278, 364

Oliva, E.,Origlia, L.,Kotilainen, J. K., \& Moorwood, A. F. M. 1995, A\&A, 301, 550

Pence, W. D. 1981, ApJ, 247, 473

Prada, F. 1995, PhD Thesis, Queens University of Belfast

Prada, F.,Manchado, A.,Canzian, B.,Peletier, R. F., McKeith, C. D., \& Beckman,J. E., 1996, ApJ, 458, 537

Prada, F.,Gutiérrez, C. M., Peletier, R. F., \& McKeith, C. D. 1996, ApJ, 463, L9

Puxley, P. J., \& Brand,P. W. J. L. 1995, MNRAS, 274,L77 
Rieke, G. H.,Lebofsky, M. J.,Thompson, R. I.,Low, F. J., \& Tokunaga, A. T. 1980, ApJ, 238, 24

Rieke, G. H.,Lebofsky, M. J., \& Walker, C. E. 1988, ApJ, 325, 679

Sams, B.,Genzel, R.,Eckart, A.,Tacconi-Garman, L., Hofmann, R. 1994, 430, L33

Sandage, A., \& Tammann, G. A. 1975, ApJ, 196, 313

Schulz, H., \& Wegner,G. 1992, A\&A, 266, 167

Scoville, N. Z.,Soifer, B. T.,Neugebauer, G.,Young, J. S., Matthews, K., \& Yerka, J. 1985, ApJ, 289, 129

Terlevich, E.,Diaz, A. I., \& Terlevich, R. 1990, MNRAS, 242, 271

Ulrich, M-H. 1978, ApJ, 219, 424 


\section{FIGURE CAPTIONS}

Fig. 1.- The optical gaseous position-velocity curves along the major axis of NGC 253 , for the H $\alpha$ (bottom), [NII] $\lambda 6584 \AA$ (middle), and the [SII] $\lambda \lambda 6717,6731 \AA$ (top) emission lines. In the middle pannel the relative intensity of the continuum around $6500 \AA$ along the major axis is also plotted.

Fig. 2.- A comparison among the position-velocity curves along the major axis of NGC 253 of the [SIII] $\lambda 9069 \AA$, the near-IR $\mathrm{H}_{2} \lambda=2.1218 \mu \mathrm{m}$ determined by Prada et al. (1996), and the measurements by Anantharamaiah \& Goss (1996) using the radiorecombination line H92 $\alpha$. The dashed lines represents the relative intensity of the continuum around $8750 \AA$.

Fig. 3.- The position-velocity curve along the minor axis of NGC 253 for our [SIII] $\lambda 9069 \AA$ data,the $\mathrm{H} \alpha$ data by Ulrich (U78) and Heckman et al. (HAX90), and the H92 $\alpha$ by Anantharamaiah \& Goss (1996). The central region is plotted magnified on the right. When present, the filled symbols refer to the fainter component. The circles in the magnified plot represent a single Gaussian fit to the [SIII] emission lines.

Fig. 4. - The velocity dispersion curve along the major axis of NGC 253 for the H $\alpha$ (top) and the [SIII] $\lambda$ $9069 \AA$ (bottom) emission lines.

Fig. 5. - The spectra of NGC 253 along the major axis in several positions in a region centred at $5200 \AA$. The main absorption line is the MgIb features. The emision at $\sim 5200 \AA$ corresponds to the $[\mathrm{NI}]$ doublet gaseous line. The heavy lines show the reconvolved spectra obtained using our algorithm (see the text).

Fig. 6. - The same than in the previous plot but in the region of the CaII IR absorption triplet $(\sim 8500 \AA)$. Only the two CaII features at shorter wavelengths (heavy lines) were used to determine the LOSVD.

Fig. 7.- The same than in the previous plot but the CaII velocities along the minor axis.

Fig. 8.- Heliocentric radial velocities along the major axis of NGC 253 obtained from the MgIb (bottom), and the CaII IR triplet (top) absorption features using the method described in the text. The solid lines represent a circular motion fit to the caII data and a linear fit to the MgI data respectively. The dashed lines represent the relative intensity of the continuum at $\sim 5000$ and 8750 Årespectively.

Fig. 9.- The same than the previous figure but for the CaII data along the minor axis.

Fig. 10.- The stellar LOSVD along the minor axis in the central regions of NGC 253. Note a certain degree 
of assymetry towards the blueshift.

Fig. 11. - The stellar velocity dispersion along the major and minor axis of NGC 253 (top and bottom respectively). The dispersions were obtained according the LOSVD determined from the CaII absorption features (August 1996 run).

Fig. 12.- A comparison between the velocity-position curves for the gas (thin line) and stars (thick line) along the major axis of NGC 253 obtained from the analysis of the $\mathrm{H} \alpha$ and CaII lines respectively. The dashed line is a linear fit to the MgIb stellar curve. The zero position in the spatial axis corresponds to the dynamical center.

Fig. 13. - The enclosed mass in NGC 253 as a function of the radius. The points and the error bars represent an estimate assuming that the velocity dispersion accounts for all the gravitational support. The solid line is a linear fits. 
Table 1: Observations

\begin{tabular}{lccccc}
\hline Date & Exposure time $(\mathrm{s})$ & P.A. $\left({ }^{\circ}\right)$ & Spectral range $(\AA)$ & Main features & Template stars (Spectral Type) \\
\hline November 95 & 1800 & 52 & $8300-9100$ & CaII, [SIII] $\lambda$ 9069 $\AA$ & HR 218 (K0 III) \\
November 95 & 1800 & 52 & $4800-5600$ & MgIb, H $\beta,[0 I I I] \lambda 5007 \AA$ & HR 218 (K0 III) \\
November 95 & 1800 & 52 & $6100-6900$ & H $\alpha,[\mathrm{NII}] \lambda 6584 \AA,[\mathrm{SII}] \lambda 67176731 \AA$ & HR 218 (K0 III) \\
July 96 & 1800 & 321 & $8300-9100$ & CaII, [SIII] $\lambda 9069 \AA$ & HD 213947 (K4 III) \\
August 96 & 1800 & 52 & $8300-9100$ & CaII, [SIII] $\lambda 9069 \AA$ & HD 4388 (G5III) \\
\hline
\end{tabular}




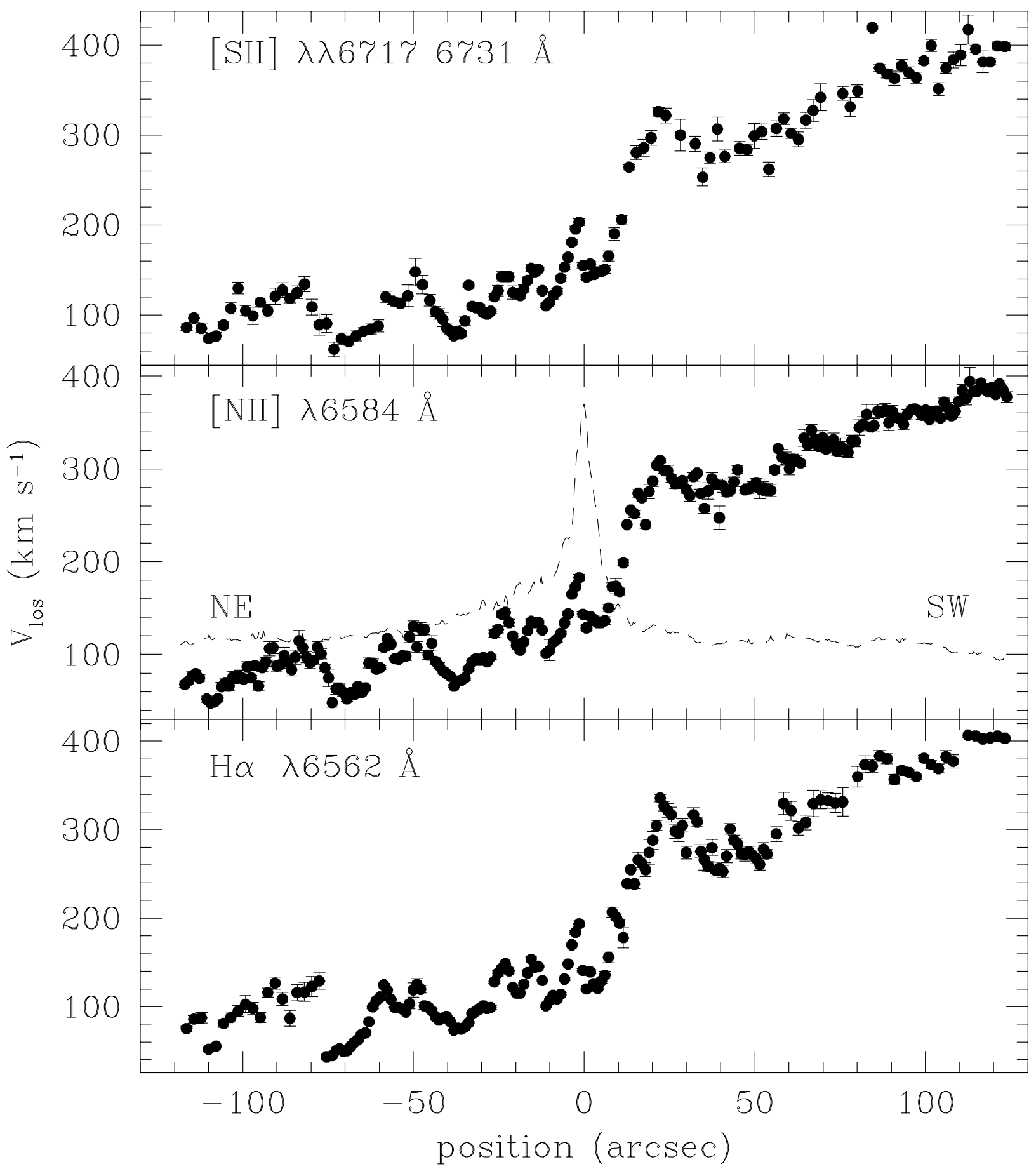




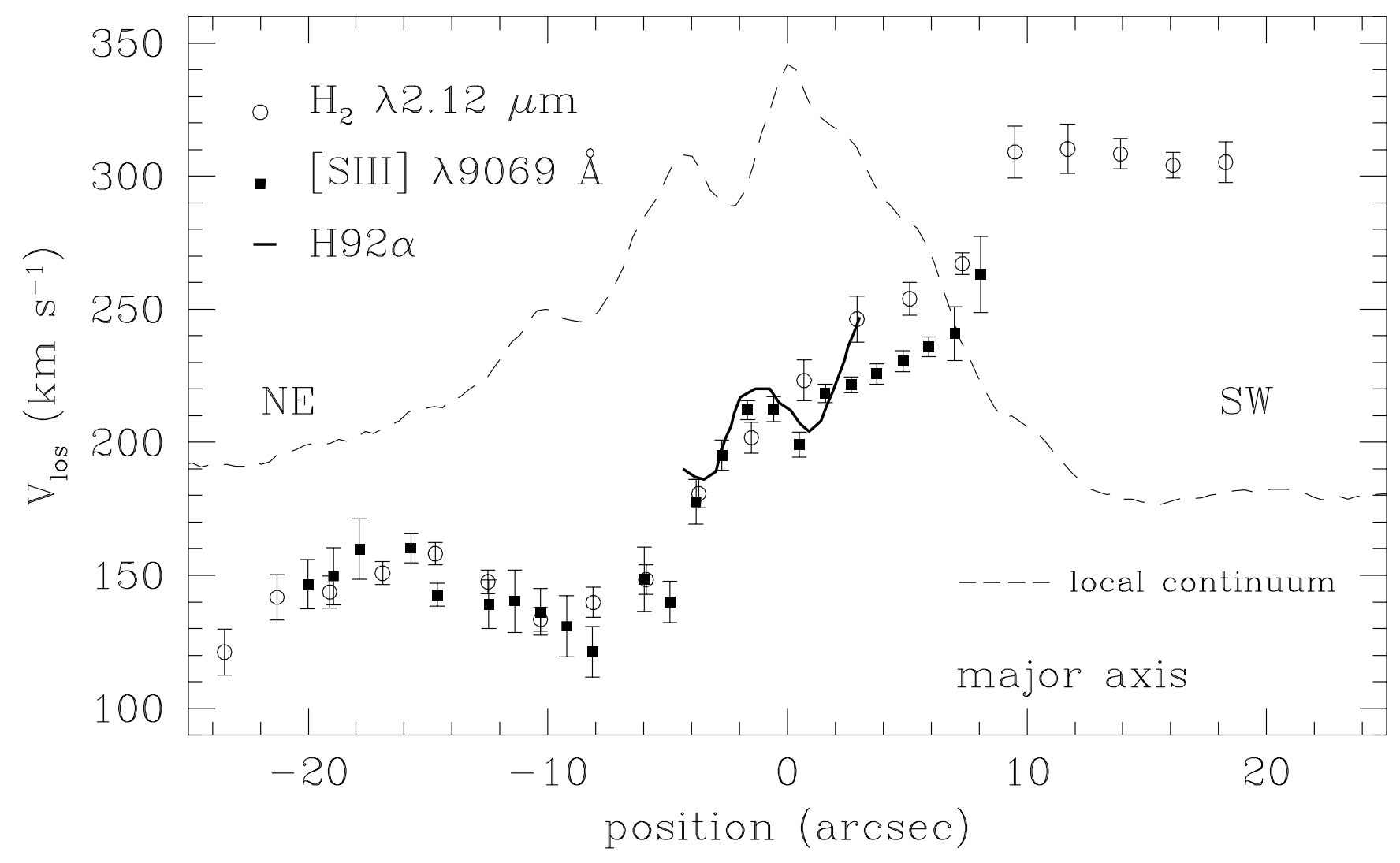




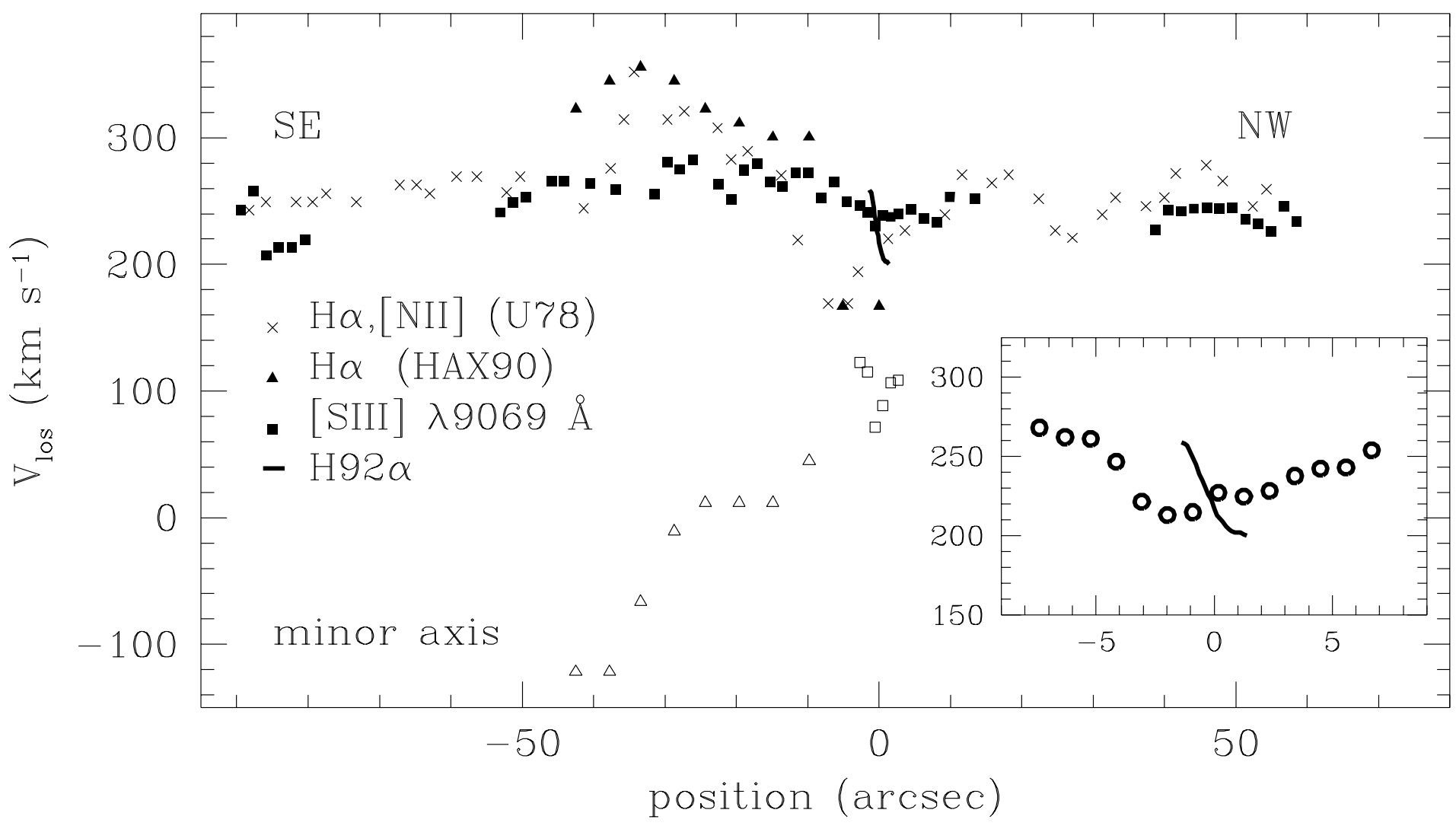



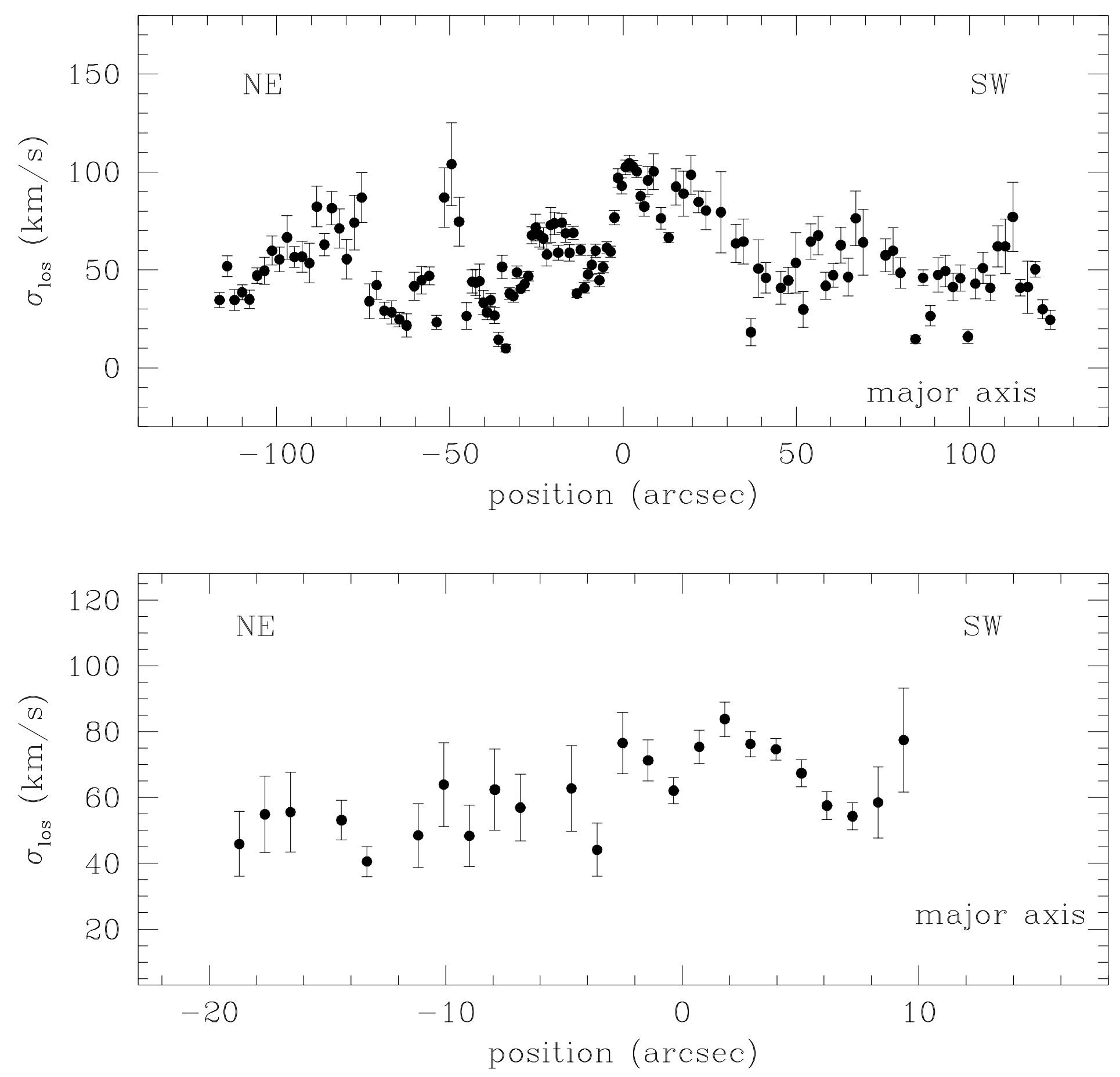

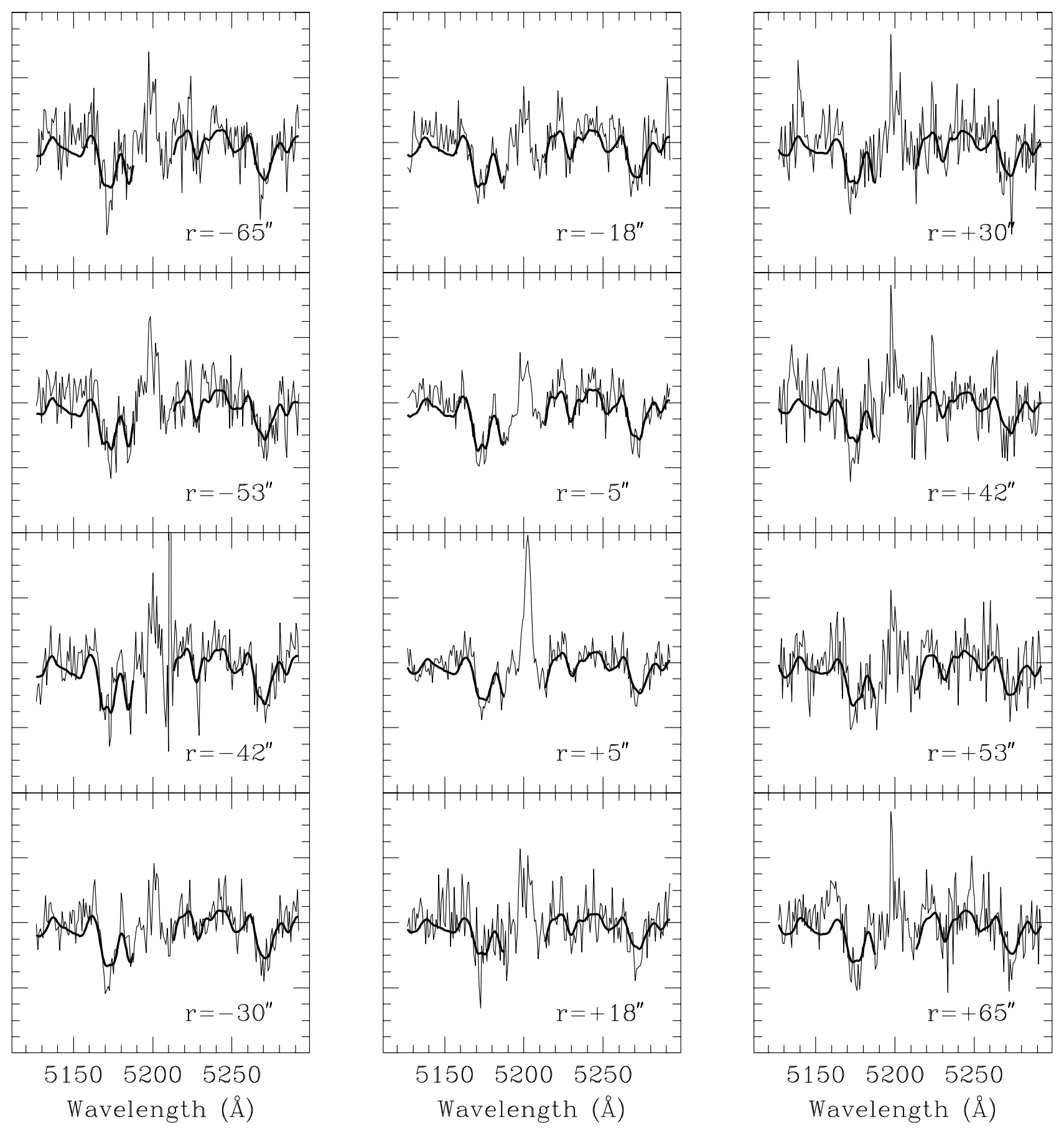

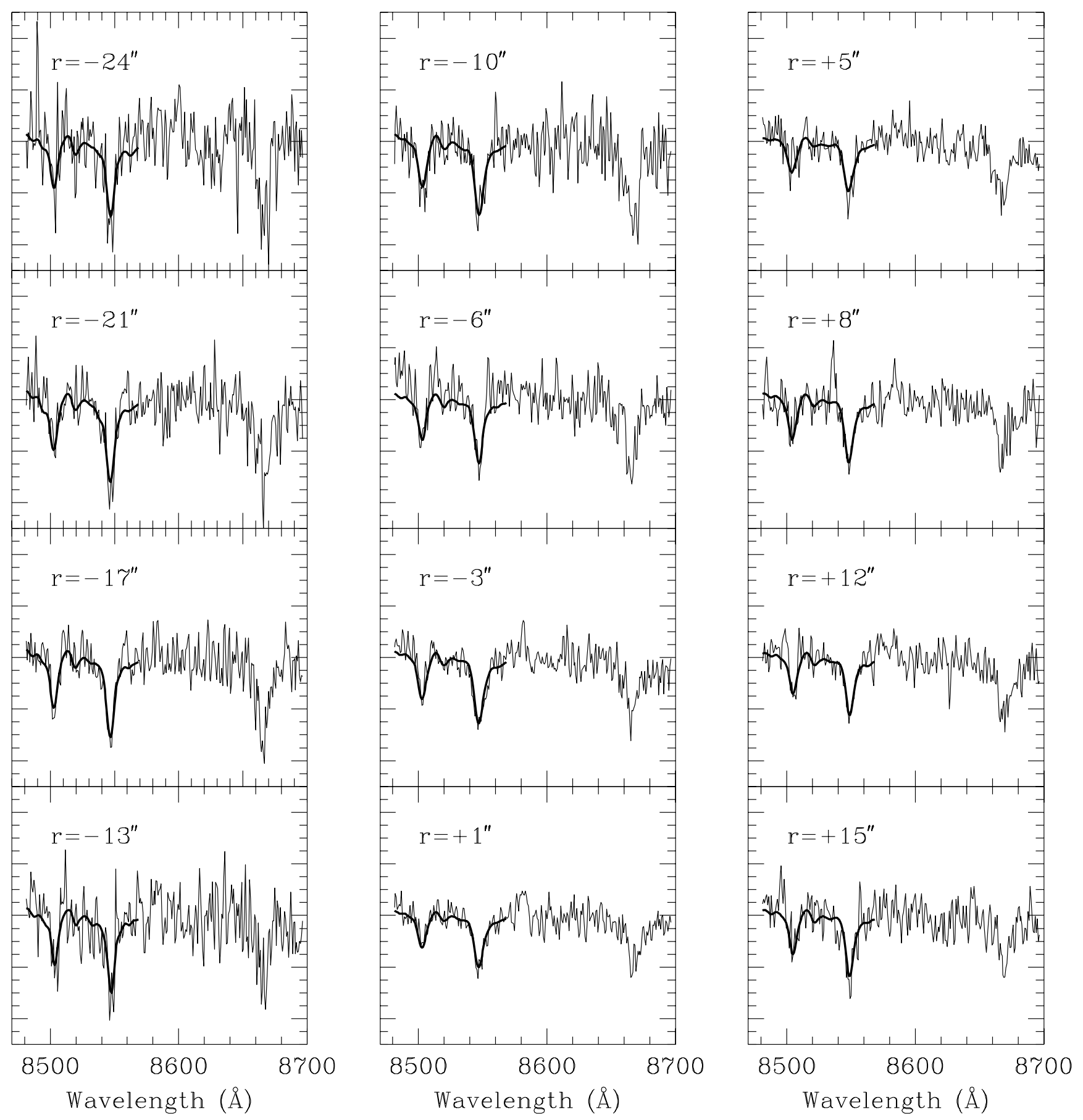

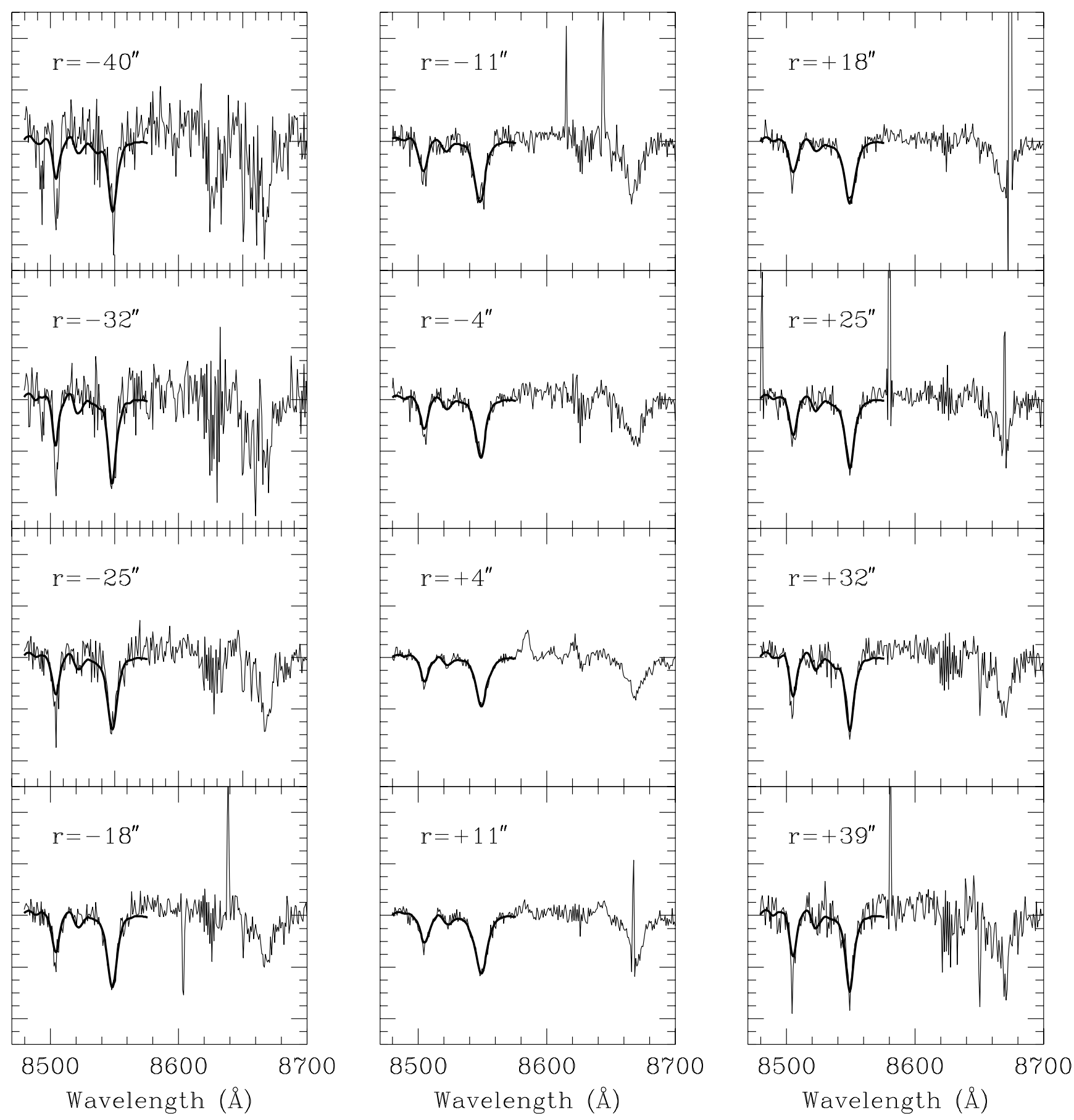

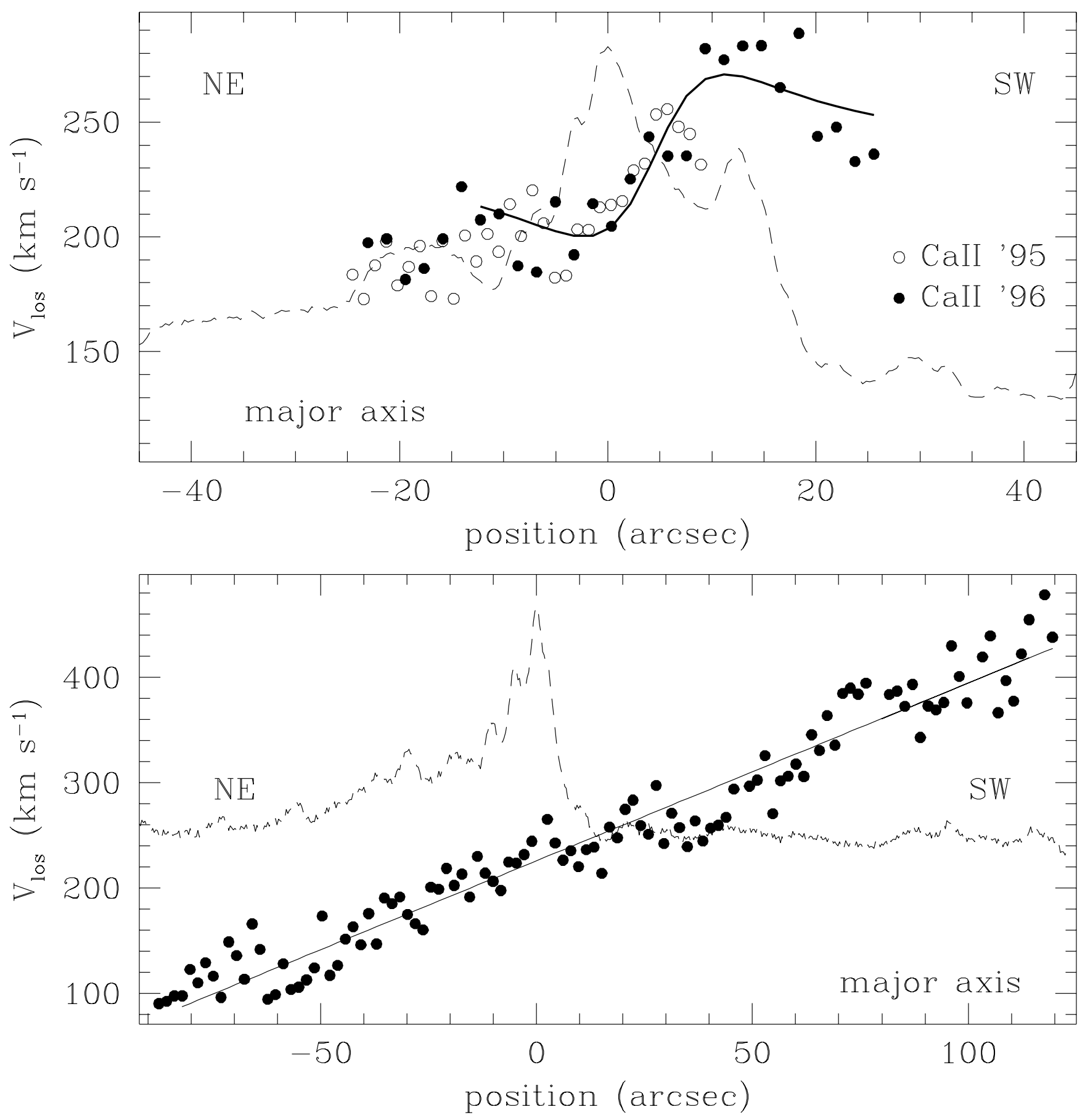


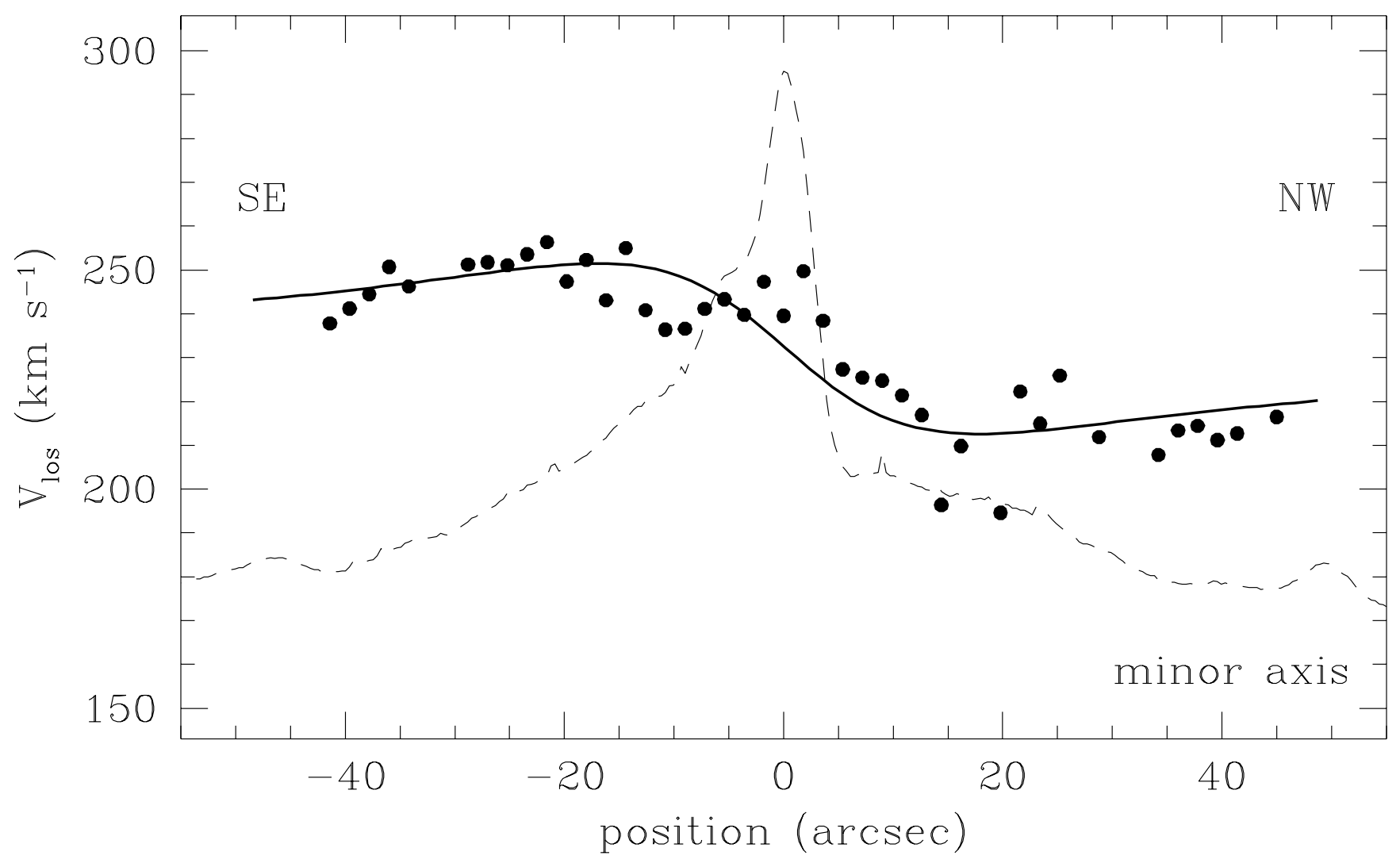




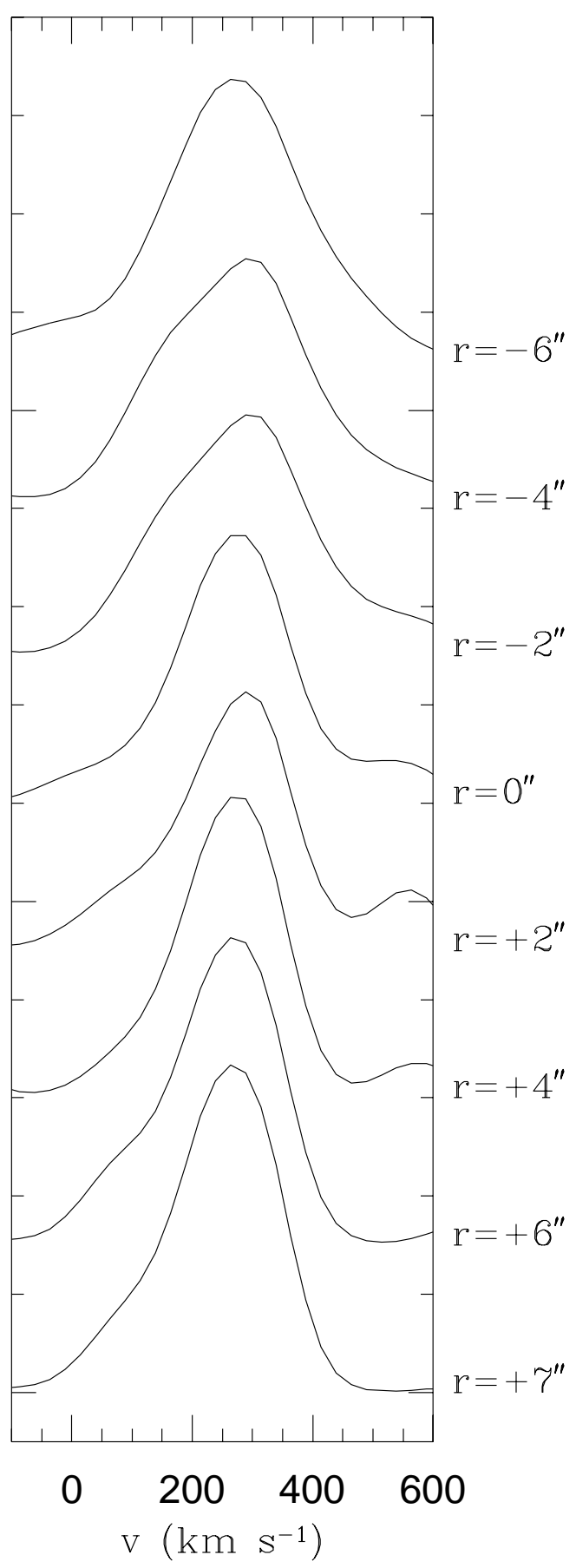



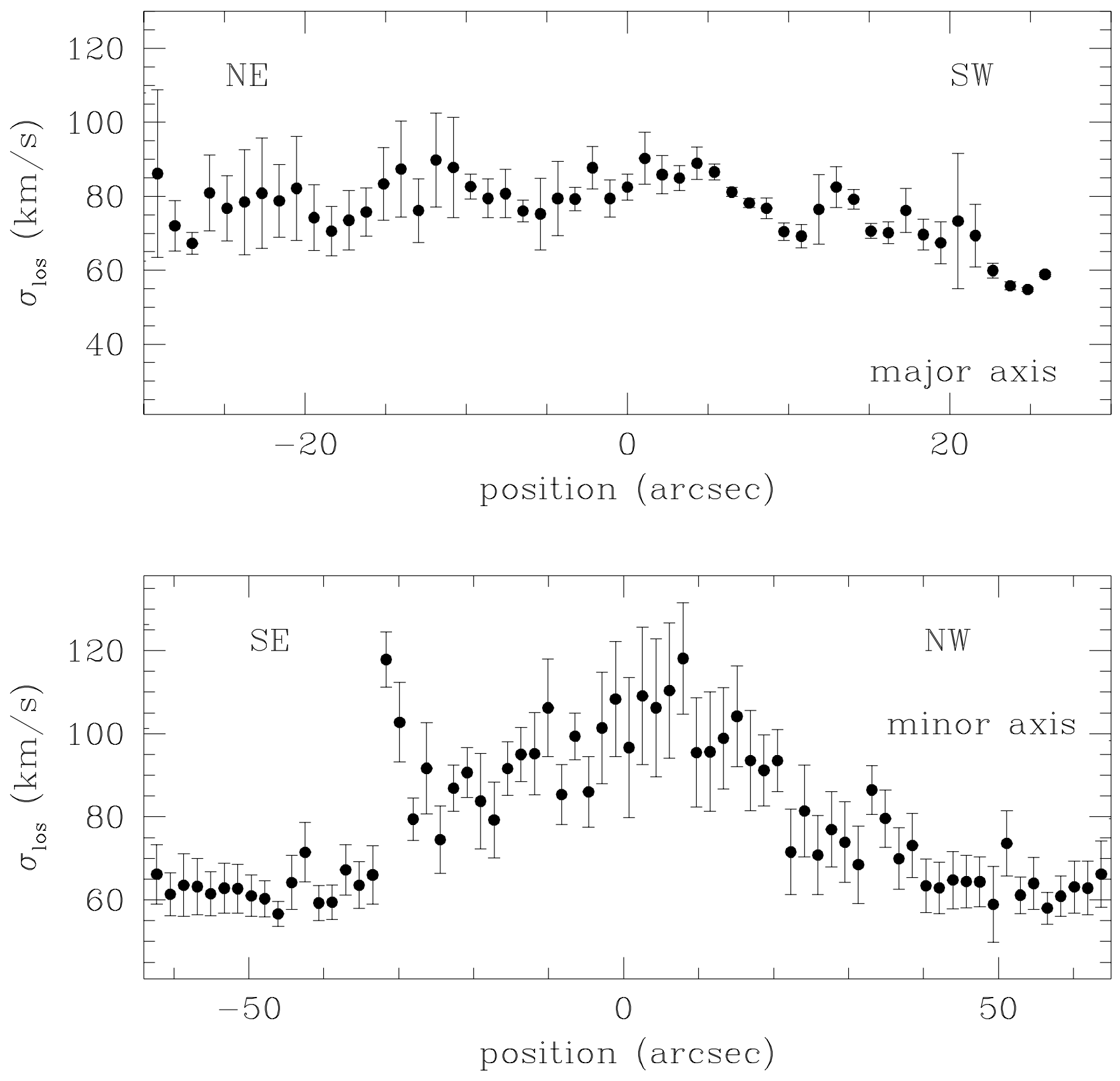


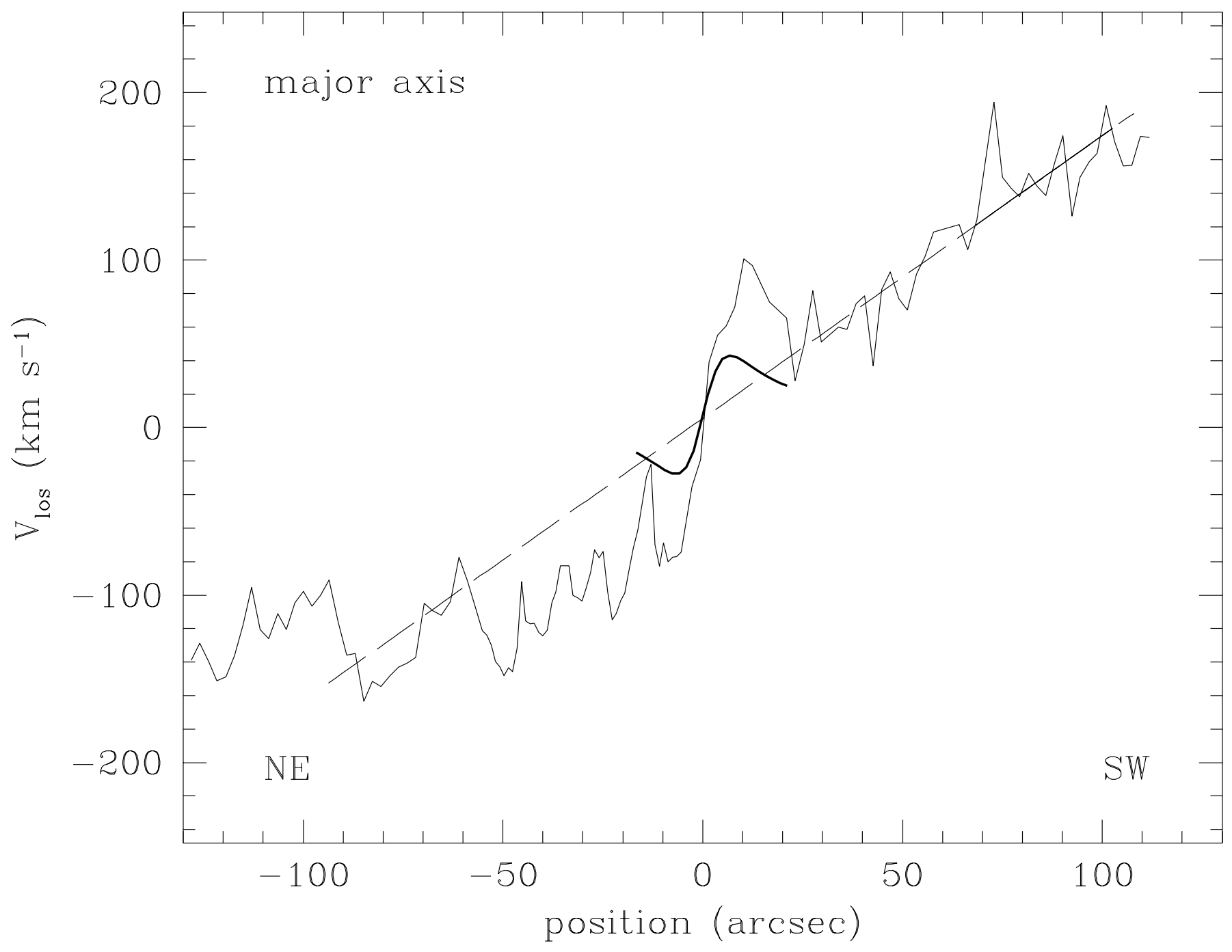




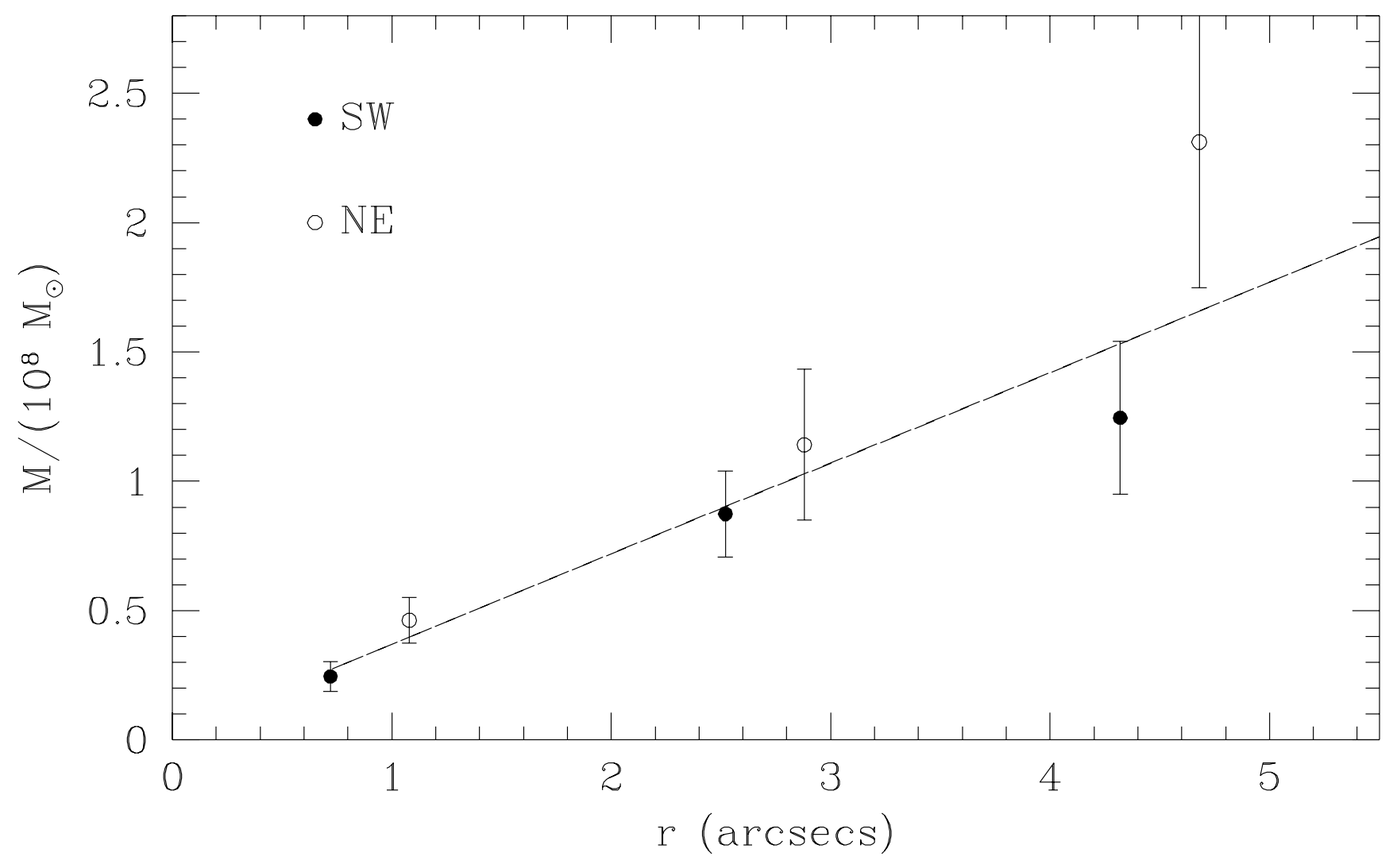

